\title{
Effectiveness and Comparison of Digital Substations Over Conventional Substations
}

\author{
Aashir Waleed", ${ }^{*}$, Umar Siddique Virk², Muhammad Tanveer Riaz ${ }^{2}$, Shaikh Bilal Mehmood ${ }^{1}$, Saeed Ahmad ${ }^{2}$, Muhammad Rameez \\ Javed $^{1}$, Ali Raza ${ }^{1}$ \\ ${ }^{1}$ Department of Electrical Engineering, University of Engineering \& Technology, Lahore (Faisalabad Campus), 38000, Pakistan \\ ${ }^{2}$ Department of Mechatronics \& Control Engineering, University of Engineering \& Technology, Lahore (Faisalabad Campus), 38000, \\ Pakistan
}

\begin{tabular}{l} 
A R T I C L E I N F O \\
\hline Article history: \\
Received: 06 May, 2019 \\
Accepted: 07 August, 2019 \\
Online: 16 August, 2019 \\
\hline Keywords: \\
Digital substations \\
Intelligent electronic device \\
Smart grids \\
Grid topology \\
Substation
\end{tabular}

\section{Introduction}

The existing grid infrastructure seems to meet part of the expectations (from traditional and renewable generation to transport and part of the distribution), but it must improve significantly from the end user and the functionalities expected from it. The forecasts indicate moderate growth in demand, a substantial increase in renewable energies and a need for firm and flexible power [1]. Consequently, a new concept of an electrical network, i.e., "intelligent network" emerges. The basic definition of "intelligent networks" may correspond to "Electric networks that intelligently integrate the behavior and actions of all the actors connected to them (those who generate electricity, those who

\footnotetext{
${ }^{*}$ Dr. Aashir Waleed, Department of Electrical Engineering, University of Engineering \&
} Technology, Lahore (Faisalabad Campus), 38000, Pakistan, Email:aashir.walid@uet.edu.pk consume it and who perform both actions) to provide a safe, economical and sustainable electricity supply"[2]. In such networks, very advanced integrated systems are used in the process of supplying electricity at all levels. The function of these integrated systems is to protect the components of the electrical system, control of the power flow and the monitoring of the process. For example, electrical system automation devices are integrated into communication networks to exchange information between various devices, as well as with supervisory systems. The technological trends of the integrated systems in the automation of electrical systems will be determined by three different technological trends such as electronic Integration, integration of switching equipment and data communication [3]. 

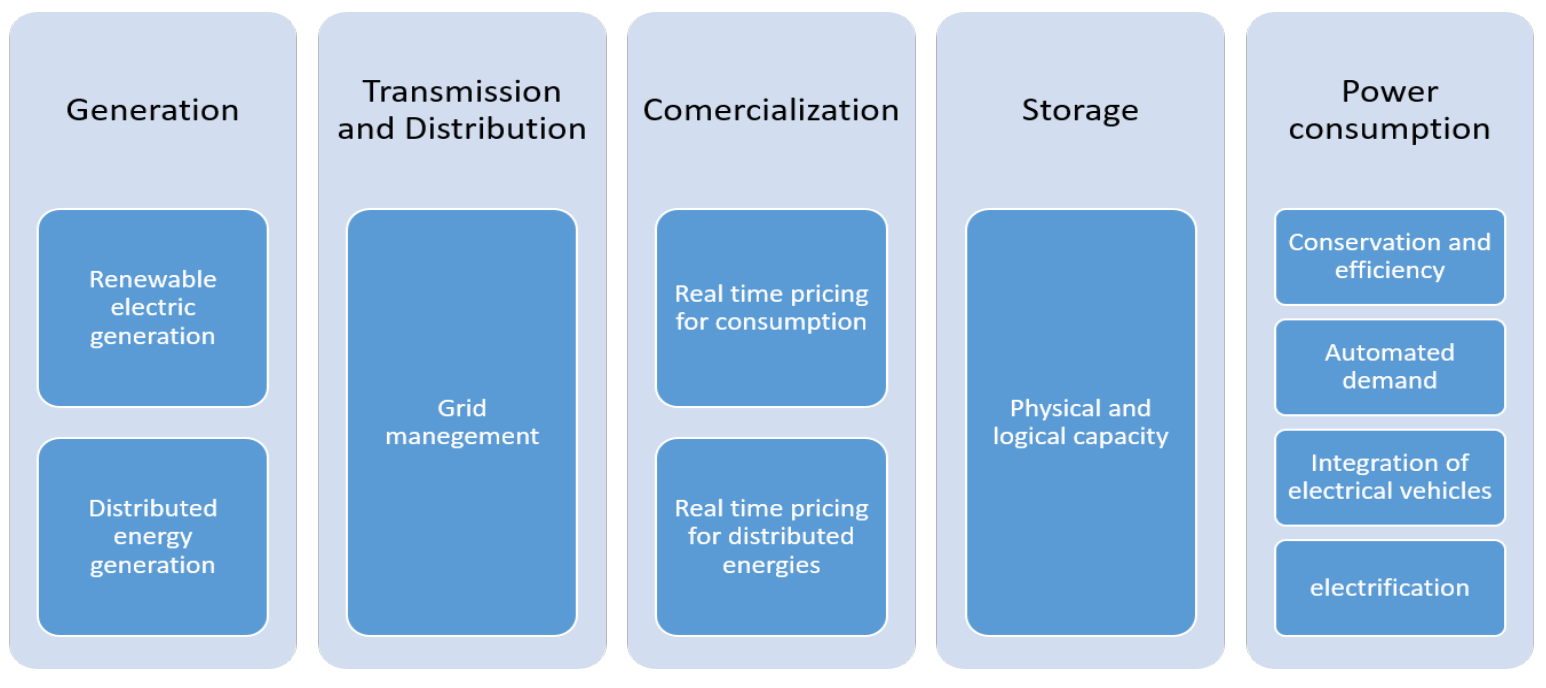

Figure 1. Challenges for current grid topology.

In the last years, an extensive research of energy production based on fossil energy sources has been performed. Fossil fuel sources have been exploited believing that there is an infinite availability of resources, but these sources cause huge damage to environment. The old energy model based on fossil fuel energy involved a chain of processes like generation, distribution, transportation, and consumption. However, to meet the consumer demand, it is necessary to upgrade the above energy model to distributed energy generation. This new energy model (Figure 1) will support variety of energy sources and an increase in the use of renewable energy and efficiency.

The main advantage of the recent energy model is distributed energy generation system. This system allows individuals to generate energy at micro-level and link to the national grid [4], which drastically reduces losses caused by the energy transport. This system facilitates the connections of all kinds of renewable energies to the system, which will ultimately improve the power system. This is possible by promoting the integration of nonmanageable energies such as wind or solar power and by using energy storage devices that will be able to charge and discharge in the network. Keeping in mind the future growth and energy trends, the future projection of digital substations is provided in Figure 2.

\section{Future of Digital Substation Automation}

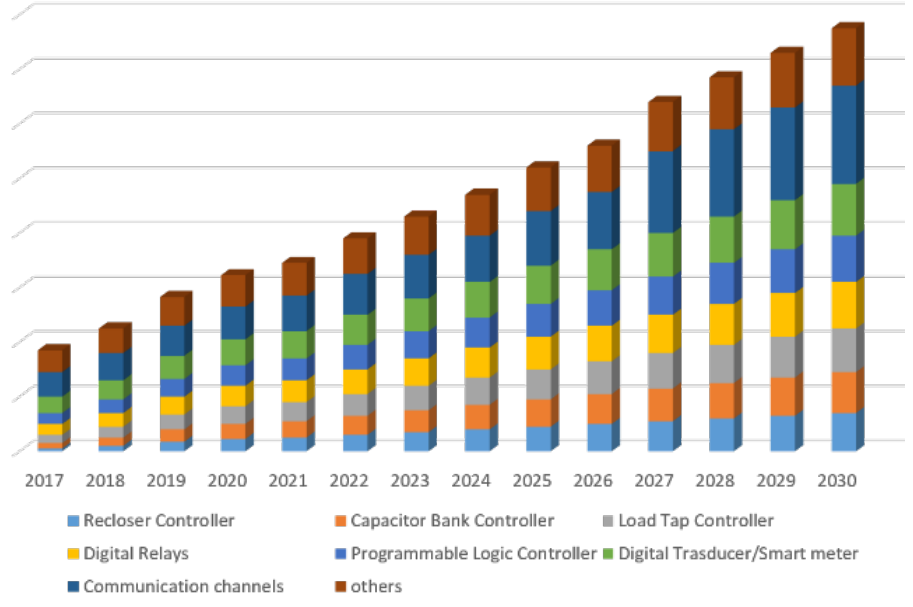

To carry out all the actions mentioned previously, the grid topology must [5], [6] have following features.

- Allow self-management of incidents, to deal with the defects that have occurred in the system and guarantee the electric flow in all the points.

- Be equipped with strength against attacks and destabilizations.

- Increase the flow of consumer participation, promoting the local generation process and providing excess energy to the network during peak hours.

- Have the capacity to supply power of adequate quality to the digital era. The higher number of generation points will allow the delivery of different energy qualities for each type of application.

- Fix a variety of generation and storage modes, thanks to the micro-grids and the generation of the energy that is distributed.

- Facilitate the flowering of markets, due to the inclusion of new elements in the network such as the electric vehicle, a more sizeable number of renewable energies, etc.

- Perform a more competent optimization of your assets and operation; this is achieved by automating all the elements that are involved.

\section{Composition of Smart Grids}

The Smart Grid concept refers to intelligent management of electricity using computer and communication technologies. The smart grid concept encompasses many interconnections between the different elements of the generation, transmission and distribution of electricity. In addition, the concept is strongly linked to the inclusion of unconventional renewable energies (both large-scale with large generators and household level) in the electricity grid, thus changing the current paradigm of uniqueness in generation towards a distributed generation concept.

Smart grid is smart energy network that integrates all the parameters of generation, transmission, distribution and 
consumption. It also provides advanced control processes to achieve an efficient and sustainable supply (Figure 3) [7]. The motivation behind creation of smart grid is: care for the environment, reliability and quality of supply and improvement in the operation of networks.

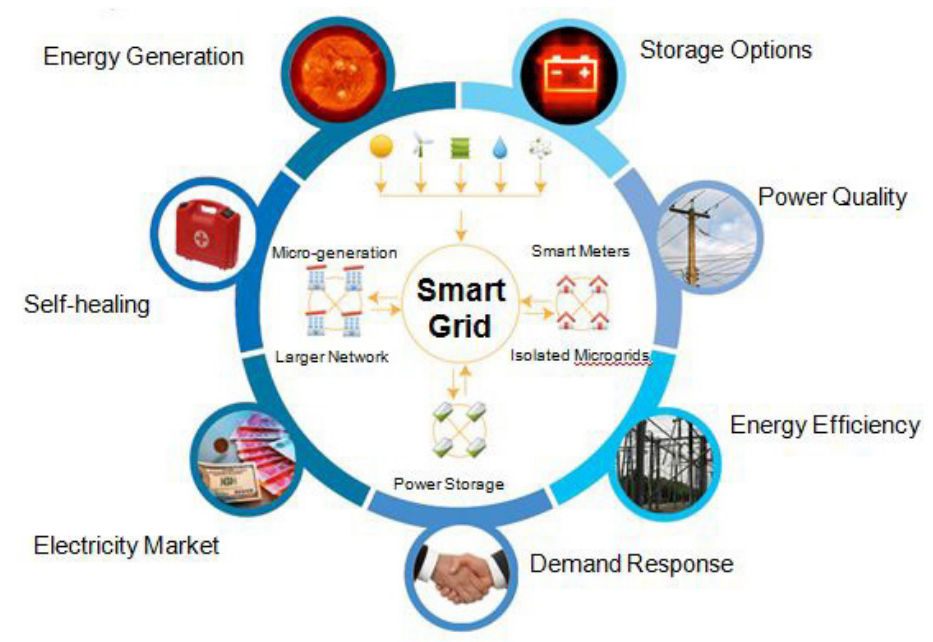

Figure 3. Different aspects that make up the smart grid

The following components must be developed to meet the above-mentioned objectives objective of smart grid:

\subsection{Smart Metering:}

The smart metering helps consumers to generate their own energy and inject surplus generated energy into the network at a certain price. This increases the dynamism of the system and decreases the prices of electricity [8].

\subsection{Load Management:}

The data delivered by the smart meters helps in effective management of load. The hourly consumption of energy as delivered by meters is the main deciding factor for the connection or disconnection management of generation sources and loads. This connection or disconnection can be automatic through intelligent controllers, which entails awareness and education of the population in general. Conclusively, this helps in developing a culture of low consumption and energy saving.

\subsection{Inclusion of Renewable Energies and Electric Mobility}

The smart grid also covers the issue of microgeneration in households. For that purpose, it is necessary to study the natural resources that can be used in the houses of different geographical sectors of the country and improve the technologies in their connection to the distribution lines.

\subsection{Renewal of The Current Transmission Networks:}

A fundamental point to achieve the above points requires the inclusion of an effective transmission network. The distributed generation and a strong increase in the amount of non-conventional renewable energies injected into the network require a transmission system that supports these bidirectional flows.
So, conclusively smart grid requires different technologies that can ensure a correct operation of this new network due to the complexity that is added to become intelligent [9].

\section{Latest Developments in Smart Protection}

Microgrid is normally operated parallel with utility grid and e.g. during faults in upstream network it can be separated quickly from utility grid and operated independently as an island grid. MMS will be responsible from the overall economic and energy effective operation of microgrid taking account the technical boundary conditions in both normal and island operation. In this paper only technical aspects related to LV microgrids are discussed. Technical choices made in the microgrid concept must be such that they can be justified by the needs of normal operation, but at the same time allowing and supporting the solutions needed

for implementation of island operation.

In overall the operation and control of LV microgrid is a very complex issue because there are number of things that will have influence to the behavior of microgrid in different ways. For example the dynamics of islanded microgrid, e.g. lack of inertia, is totally different when compared to the normal operation of the microgrid parallel with utility grid. Islanded microgrid is much more sensitive to disturbances and successful island operation requires fast, accurate and stable control.

In addition the protection of the future microgrid is a challenging issue and very strongly connected to the control and operation issues of a microgrid. The conventional protection in distribution networks is designed to operate for high fault current levels in radial networks, but during island operation of the microgrid high fault currents from the utility grid are not present. Also most of the DG units that will be connected to the LV microgrid in the future are converter interfaced and have limited fault current feeding capabilities. This means that the traditional fuse protection of LV network is no longer applicable and new protection methods must be developed. The developed protection scheme for microgrid must be supported by the technical choices made in the microgrid operation and control issues.

In the development of the new protection scheme for LV microgrids many things must be considered including amount of protection zones in LV microgrid, speed requirements for microgrid protection in different operation states and configurations and protection principles for parallel and island operation of the microgrid.

\section{Technological Trends}

Among the new technological trends of smart grids, substations are intelligent networks, which are designed to manage the consumption of each element connected to it in an "intelligent" way [10]. These networks will use innovative equipment and services, along with new communication, control, monitoring, and self-diagnosis technologies that will help to achieve the following objectives [11]:

- Reinforce and automate the network, achieving an improvement in the functionality of the system, having better quality values and less loss in the network. 


\section{A. Waleed et al. / Advances in Science, Technology and Engineering Systems Journal Vol. 4, No. 4, 431-439 (2019)}

- Optimize the connection between the areas that have renewable energy sources, improving the connection capacities and reducing connection costs.

- Study the decentralized generation architectures, making smaller installations (distributed generation) in balance with the system [12].

- Develop intermittent generation integration and new storage technologies

- To advance in the development of the electricity market, allowing new functionalities and services to the market.

- Allow consumers to manage their energy efficiency better through active demand management.

- Enable the penetration of the electric vehicle, accommodating these new mobiles and dispersed loads to the grid, minimizing the development of new infrastructure and enabling the energy storage features they possess [13].

\subsection{Advanced Automated Distribution}

Advanced distribution automation allows the electrical power distribution system to be completely controllable to increase efficiency, sustainability and reliability. Some applications for advanced automation of distribution are monitoring, data acquisition and control, voltage and reactive power control, fault localization, automatic reconfiguration, isolation and restoration service, user information, time management, asset management and remote control. The main reason for the advanced distribution automation is to guarantee the safety of the service, the quality of the energy and the efficiency of the energy system. The above is possible once the next three control processes of the distribution operation are automated [14]. These control processes are: (1) the data is arranged almost in real time, (2) the optimal decisions and (3) the coordination with the services of the different generation and distribution systems to control the distribution operations.

An automated system of advanced distribution will be able to perform data collection, along with the verification and correction of consistency of that data. Besides, the system should reconfigure the multilevel feeder. It should be pre-armed with the corrective action schemes and the organization of urgency actions in distribution. It should take care of the previous assembly of recovery schemes and coordination of corrective actions in the distribution, registration, and reports [15].

\subsection{Distributed Energy Resources}

Distributed energy source technologies consist of storage and power generation resources labeled as "behind the meter." These resources are installed at customer's end and are operated to supply partial or total energy consumed by the client [16]. These energy resources may also be able to inject energy into the distribution system and the transmission system or into a local network in parallel with the public network. Moreover, certain technologies such as combined heat and power (CHP) systems, photovoltaic solar energy (PV), microgrids, wind turbines, microturbines, standby generators and energy storage are included [17]. These resources represent immense importance because they can contribute to the reliability of the network. They may be able to produce energy to support the load of the host or the system. These technologies have evolved at a great pace in the years that have passed. However, the current challenges require unique planning, operation, and market management [18].

Moreover, the term that characterizes it as "behind the meter" means that it represents certain resources that are not directly connected to the commercial electric power system but are connected to the customer's access point. In this case, it is possible that the resources are acting to meet the customer's internal electrical loads, or the resources may be working to sell to the electric power system [19].

\subsection{Information and Communication Technologies}

Advancement in information and communication technologies (ICT) has been helping to maintain the distribution of electricity while limiting the environmental consequences. In addition to this, ICTs allow greater incorporation of renewable energy sources, allowing the transport of little carbon. ICT also includes electric vehicles and inducing changes in the structure with respect to excessive consumption of electricity [20]. Among its specific political implications, ICTs include convergent telecommunications services. These services can be the changes in connectivity requirements, changes in the roles of ICT companies as partners in the electricity sector and the development of new ability for personnel of the industry [21]. Smart electrical substations guarantee the interoperability between elements connected to the network. They ensure privacy to the critical infrastructures and commercial use of power-sharing applications.

The communication between different devices of the Transformation Center and the Control and Supervision Center has not yet had a standard that allows efficient data communication. This limitation makes it difficult to implement the SCADA systems in the transformation centers [22]. Moreover, the interface with the relays was in many cases non-existent. To resolve these problems, the International Electrotechnical Commission launched some standard protocols called as IEC 61850. This protocol defines the communication between different devices connected to a local area network. Intelligent Electronic Devices (IEDs) are replacing the old wired communication techniques.

IEDs can integrate communication elements for tele-control [23]. Out of the three main layers of smart grid technologies (energy layer, communication layer, and application layer), the communications layer enables the existence of the smart grid (Figure 4). Communication technologies act as a circulatory system to interconnect the different systems and devices. It links the energy layer with the application layer, to communicate both with the energy supply chain [24].

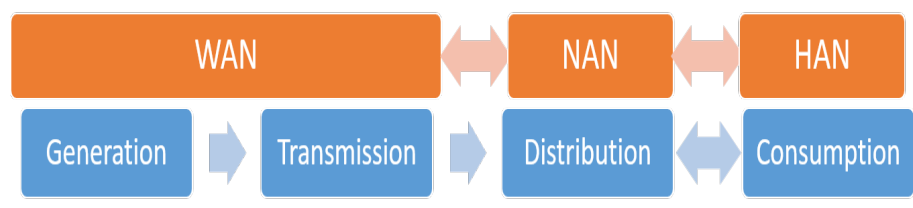

Figure 4. Smart grid topology for power flow in blue and information flow in orange 


\subsection{Supervisory System}

The components of a supervisory system range from detection equipment, fault protection, communication, measurement, and data processing by the software. The software integrates all the intelligent devices (IED) that are part of the system [25]. However, the most relevant components of a supervisory system are the sensors of state and measurement. These sensors are installed in the field and are the base of the generation information for the supervising system. The status sensors have only two possible values zero or a logical one. These logical values reflect the states of the control elements of the system. For example, the status of a power switch can be estimated by the logical values of these sensors [26]. The protection relays that are currently manufactured are mainly IEDs. A typical IED (Intelligent Electronic Device) can contain around five to twelve protection functions and five to eight control functions. Control functions include separate devices control, an "auto-recloser" function, the self-monitoring function, and the communication functions etc. [27]. Currently, some IEDs work with the standard for the automation of IEC61850 electrical substations. This standard allows interoperability and good communications capacity (Figure 5) [28].

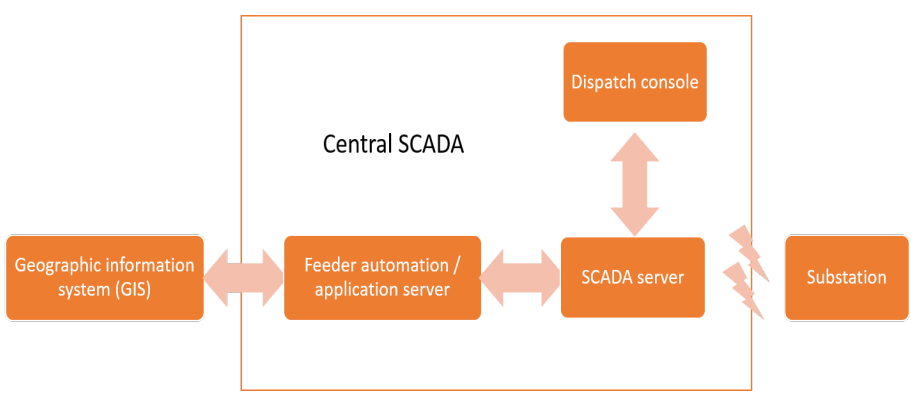

Figure 5. Feeder automation based on SCADA, current proposals in execution.

The communications network is the backbone of the entire supervisory system in real time. Its efficiency will depend on the speed and response time of the system to external events. The software is the last component that is required in the supervisory system; it is the way of representing the system [29].

\subsection{Protection and Automation of the Network}

The automation systems in substations for interconnecting a series of devices have existed for about 20 years, using proprietary protocols. These methods have been mainly responsible for the supervision of elements. Nowadays, this type of systems has evolved and continue to do so by following the protocols and actions declared in IEC 61850 [30]. This can be achieved by using peer-to-peer communications and enabling the exchange of data between systems at various levels and with various tools. This system also allows the supervision, control a series of devices or variables [31]. On the other hand, the increase in renewable energy generation and co-generation require the application of technologies in such a way that their management and protection is possible [32]. This impact on electrical networks is currently manifested, both in the transmission and distribution. The effect on substations, both in their protection and in their control, has been profound. This is due to the need of managing the intermittent electricity and several voltage levels [33].

The automation systems in substations for interconnecting a series of devices have existed for about 20 years, using proprietary protocols. These methods have been mainly responsible for the supervision of elements. Nowadays, this type of systems has evolved and continue to do so by following the protocols and actions declared in IEC 61850. This can be achieved by using peerto-peer communications and enabling the exchange of data between systems at different levels and with various tools. This system also allows of supervisory control of devices or variables [34]. On the other hand, the increase in renewable energy generation and co-generation require the application of technologies in such a way that their management and protection is possible. The effect on substations, both in their protection and in their control, has been profound. This is due to the need of managing the intermittent electricity and several voltage levels. Therefore, for the development and supervision of the elements of the network, there are many emerging solutions for energy monitoring and parameters associated with it [35].

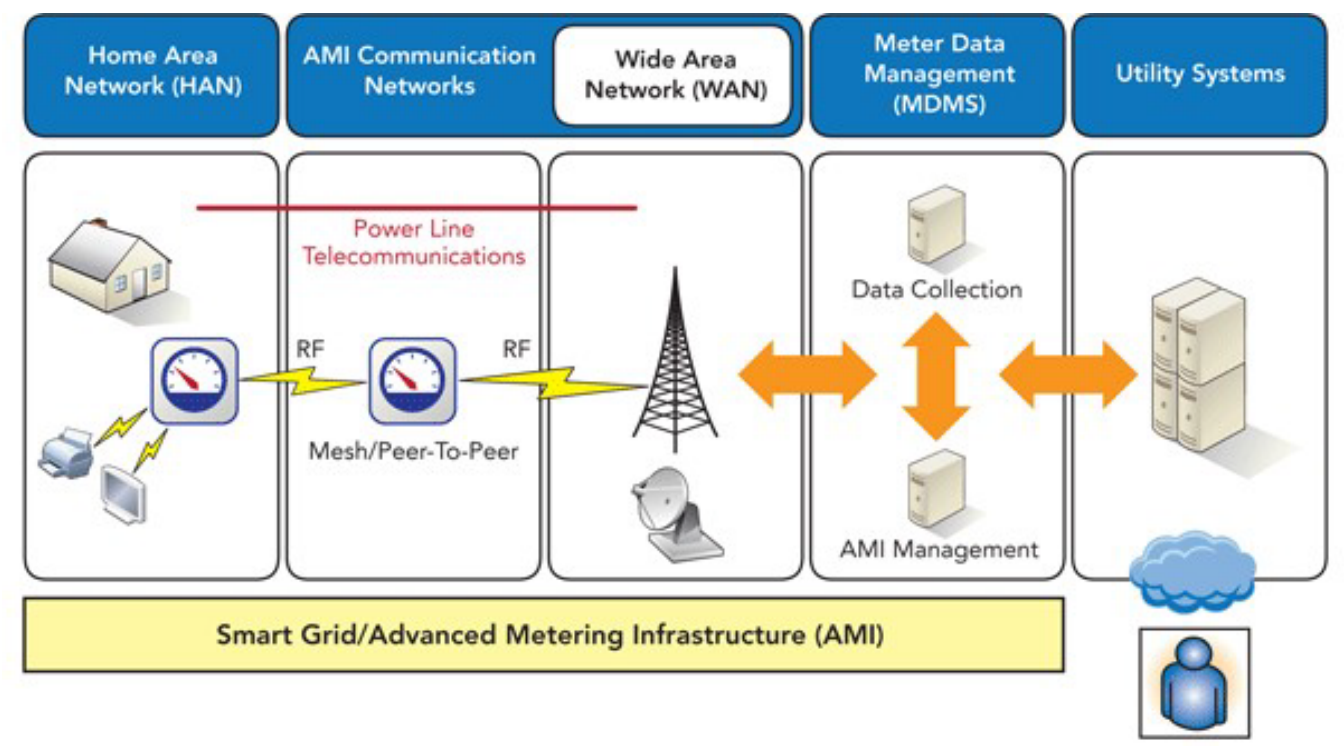




\section{Digital Substations Control}

To carry out and be able to develop all the functionalities that are expected from smart grids, this capacity (primarily redundant bi-directional data communications and the possibility of remote management) must be reproduced throughout the entire distribution network. It is already beginning to work in the section of the electrical consumption measurement infrastructure [36]. It must be implemented and improved in all sections of the power system.

The systems are based on bidirectional communications, technologies are associated with RF (radio frequency) wireless communications or power line carrier communications or broadband options (Figure 6) [37].

The desired functionality of smart meters could be the bidirectional communications with the electric company and with other devices (such as possible local energy managers). The other possible use of smart meter is alarms associated with quality of supply, personalization of the contract, planning, and possibility of bidding and purchase of electricity at the desired moments and control of loads, etc. [38]. This communication and monitoring capacity must be expanded to all measurement points and equipment in the electrical infrastructure. Also, it should be able to manage all distributed energy resources, considering not only the amount of energy flow but also the direction of it.

The technologies that affect smart grids are those that try to optimize and rationalize the management of electricity demand. As already mentioned, electrical energy cannot be stored (at least in enormous quantities), so it is necessary to generate it at the same time as it is needed. This situation means that at the very moment when a dedicated appliance is turned on, there must be a power plant (or any other type of electricity generator) that produces the necessary electricity [39].

\subsection{Transport Network}

To guarantee the electrical supply, in the absence of a line, the topology can be reconfigured to redirect the energy flows and thus avoid cutting off the supply. In this case, the systems of fault detection and reconfiguration of the network must act in small time spans for the control to be carried out in real time.

\subsection{Distribution Network}

The trend towards Smart Grids implies a replication or expansion of the existing capacities in the transport network to the distribution network. Considering that, unlike the conventional transport network, there are several owners and managers of the distribution networks. This situation will involve the definition of standard instruments and the creation of tools based on proven technology solutions. These tools will allow the integration of all types of generating plants, decentralized management of energy and automation of distribution and measurement services, supported by a system of communications that reaches the end user [40].

The electrical distribution companies can analyze and remotely control the electrical network by replacing meters with smart equipment. This equipment can take hourly measurements with Tele-management capacity [41]. In most cases, to guarantee interoperability, it is likely to install concentrators in the transformation centers with communication capabilities to control center of the corresponding distributor [42]. On the contrary, for the communication between the transformation center and the meters, the tendency in Europe is to use the power line carrier. The evolution of current electricity grids towards intelligent electricity networks implies the start of new intelligent equipment. This equipment will have local decision-making capacity and new communication technologies or the adaptation of existing ones. The current map of communication protocols used in the electrical system is very extensive and is regulated by the TC57 technical committee of the International Electrotechnical Commission (IEC). Thus, IECTC57 brings together several working groups to standardize the communications in the electrical system through the development of data models and generic interfaces. Each of these working groups has been responsible for defining and maintaining a communication standard based on the communication needs of each point of the electrical network [42].

Thus, it is worth highlighting:

- IEC60870-5 to communicate SCADA masters and electrical substations for the control and acquisition of data on serial lines or TPC / IP

- IEC60870-6, also known as TASE-2 for communications between powers centers over WAN networks.

- IEC61970 to interconnect energy management applications or EMSs in the environment of the control centers.

- EC61968 to communicate the control centers with the systems of the distribution network.

- IEC61334 for communications on PLC distribution lines.

- IEC62325 that defines a new interface between local utilities and the liberalized energy market.

- IEC62351 to determine security profiles to be used in all the previous ones at the TCP / MMS / 61850 level.

- IEC61850 (see fig 5) for automation in the environment of electrical substations (station and process buses) and communication between their IEDs (Intelligent Electronic Devices).

With the reference IEC61850 (see Figure 7 [43])), other similar regulations have been developed in other areas of application [44] such as:

- IEC61400-25 inherits a subset of communications services defined in IEC61850. It provides a new mapping of communications to Web Services and extends the data model modeling the functionalities, data, and attributes present in a wind turbine.

- IEC61850-7-420, which extends the data model by modeling the features, data, and attributes present in distributed generation systems such as photovoltaic systems, storage systems, diesel generators and heat exchange systems.

- IEC61850-7-410 is identical to IEC61850-7-420 for hydroelectric power plants. 


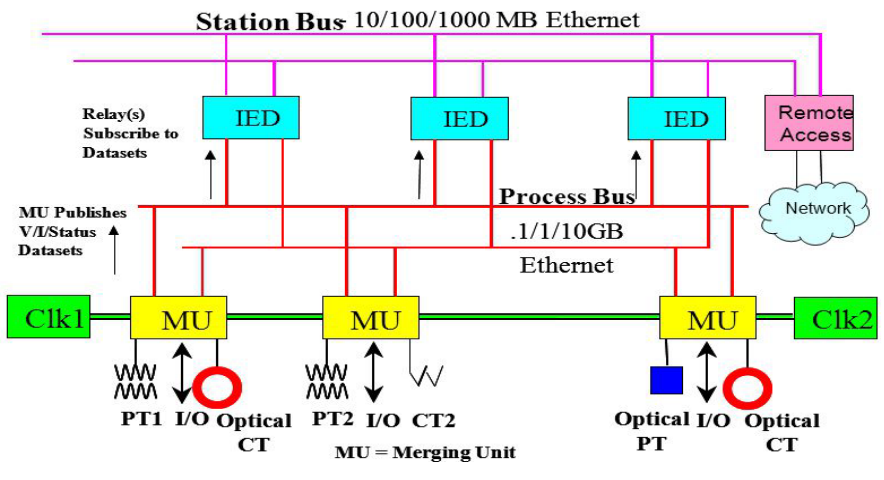

Figure 7. IEC61850 substation architecture.

\subsection{Less Copper}

Digital Substations utilize less copper by (1) replacing copper cables between switchyard and relay house by fiber optics, (2) replacing horizontal wiring between protection and control IEDs with IEC 61850, (3) reducing the number of connections between primary apparatus and redundant process interface modules and (4) reduction in cables for power supply and other connection for primary equipment.

\subsection{Less Transport}

In digital substation, more than 30 tons of material can be saved [45]. This helps in less transportation. The fiber optic installation reduces the cabling under $90 \%$ and by using optical instead of conventional CTs achieves an $80 \%$ weight reduction on CTs.

\subsection{Space Reduction}

IED installations require less space than conventional IOs. A space reduction around $30 \%$ to $60 \%$ for panels is possible. This results in higher integration of control and protection systems and reduction of switchyard footprint.

\subsection{Less Installation and Outage Time}

Digital substations cause faster installation of control systems due to reduction in number of panels for installation. There are fewer cables to be pulled, connected and tested. This causes reduction of feeder outage time by 40 to $50 \%$ during secondary system upgrades. This helps in full system test from process IO to protection, control and SCADA system offsite and Installation of new FO based system while the station is in service

\subsection{Operational Cost Reduction}

The Supervision of all exchanged data reduces the need for periodic maintenance testing and permanent supervision enables fast and precise actions in case of failures. IEC 61850 testing and simulation features enable fast and save isolation and testing of protection functions. Standard compliance enables efficient future retrofits of the secondary system (see Figure 5).

\section{Conclusions and Recommendations}

This work provides a review on digital substations importance and their working. This work emphasized on the importance of digital substations along with latest trends in modern smart grids. The provided comparison study proved the effectiveness of modern digital grids over conventional grids as well.

Apart of above-mentioned benefits, digital substations in Smart Grids provide a series of direct advantages to energy companies and end users that will help in the development of the energy market. The evolution of the electricity network provides the distribution companies with following benefits:

- Reduction of energy losses.

- Efficiency.

- Accomplish the optimization of the network infrastructure.

- Offering a better service to the client, with more commercial advantages (new rates, payment for use, etc.).

The users get the following benefits:

- Pay per use.

- Flexible rates.

- Remote management of the power supply.

Although at present, the home automation sector is in a complicated situation. It is promoting the instillation of control and automation systems in the home aimed at increasing energy efficiency, reducing energy losses, generated energy integration and accessibility to people with disabilities [46]. Currently, the number of agents involved in the electricity system has increased due to the liberalization of the energy market. The appearance of new participants is expected such as energy companies, system integrators, auditors, etc. It also predicts the development of renewable energies e.g., efficient solar cells, wind powered systems [47]-[48] and electric vehicles exponentially, in parallel with Smart Grids. So, it is necessary to keep these concepts in mind when carrying out any research or development related to the evolution of the electrical network.

$\mathrm{R} \& \mathrm{D}$ in the field of telecommunications for the development of new communication techniques and optimization of existing ones is still in progress. Technologies such as PLC, wireless connections or fiber optic, will provide a fundamental part of the Electrical Network of the future. Development in areas of power electronics, energy transport, electrical storage, energy efficiency, automated systems, new intelligent control techniques, etc. needs more investment and research [49]. Insofar, smart grids would play a great role in the development of smarter world having smart cities, smart homes, smart vehicles, etc. The ultimate requirement is to create systems of objects that are configured automatically and can operate autonomously [50]-[53].

\section{Conflict of Interest}

The authors declare no conflict of interest.

\section{Acknowledgment}

We would like to thank Dr. Muhammad Nasir for his assistance on data organization and guidance for writing.

\section{References}

[1] Ramie, J. 2017 "Testing requirements for intelligent electronic devices in transmission \& distribution facilities"in IEEE International Symposium on Electromagnetic Compatibility \& Signal/Power Integrity (EMCSI), 7-11 Aug 2017: 1-36, Washington, DC: IEEE. 


\section{A. Waleed et al. / Advances in Science, Technology and Engineering Systems Journal Vol. 4, No. 4, 431-439 (2019)}

[2] Salvadori, F., Gehrke, C.S., Hartmann, L.V., de Freitas, I.S., Santos, T. D., and Texeira, T.A., "Design and implementation of a flexible intelligent electronic device for smart grid applications." in IEEE Industry Applications Society Annual Meeting, 1-5 Oct 2017: 1-6, Cincinnati, OH: IEEE.

[3] Chen, J., and Fu, Q., 2016 "Design of experiment platform for digital substation based on IEC 61850." In 5th International Conference on IEEE Computer Science and Network Technology (ICCSNT), 10-11 Dec 2016: 48, Changchun: IEEE.

[4] Hu, J., and Vasilakos, A., 2016 "Energy Big Data Analytics and Security: Challenges and Opportunities." IEEE Transactions on Smart Grid 7(5): 24232436, doi:10.1109/TSG.2016.2563461.

[5] Kong, P., 2016 "Wireless Neighborhood Area Networks with QoS Support for Demand Response in Smart Grid", IEEE Transactions on Smart Grid 7(4): 1913-1923, doi: 10.1109/TSG.2015.2421991.

[6] GVR "Substation Automation Market Size, Share | Global Industry Report 2024", (Accessed: 06- Jun- 2018) https://www.grandviewresearch.com/industry-analysis/substationautomation-market/.

[7] Faheem, M., Shah, S., Butt, R., Raza, B., Anwar, M., Ashraf, M., Ngadi, M. and Gungor, V., 2018. "Smart grid communication and information technologies in the perspective of Industry 4.0: Opportunities and challenges." Computer Science Review 30: 1-30, doi: doi.org/10.1016/j.cosrev.2018.08.001.

[8] Hong, T., Chen, C., Huang, J., Lu, N., Xie, L. and Zareipour, H. 2016. “Guest Editorial Big Data Analytics for Grid Modernization." IEEE Transactions on Smart Grid 7(5): 2395-2396, doi: 10.1109/TSG.2016.2593358.

[9] Siano, P., 2014. "Demand response and smart grids-A survey." Renewable and sustainable energy reviews 30: 461-478, doi: doi.org/10.1016/j.rser.2013.10.022.

[10] Mohtashami, S., Pudjianto, D., and Strbac, G., 2017. "Strategic Distribution Network Planning with Smart Grid Technologies" IEEE Transactions on Smart Grid 8(6): 2656-2664, doi: 10.1109/TSG.2016.2533421.

[11] Noriaki, K., 2013. "Electric network innovation by digital grid." IEEJ Transactions on Power and Energy 133(2): 141-144, doi: 10.1541/ieejpes.133.141.

[12] Hayashi, K., Kato, R., Torii, R., Taoka, H., and Abe, R., 2015. "Bi-directional power flow through a digital grid router" Journal of International Council on Electrical Engineering 5(1): 42-46, doi: https://doi.org/10.1080/22348972.2015.1068511.

[13] Rahim, M., Khalid, A., Javaid, N., Alhussein, M., Aurangzeb, K., and Khan, Z., 2018. "Energy efficient smart buildings using coordination among appliances generating large data", IEEE Access: 34670-34690, doi: 10.1109/ACCESS.2018.2805849.

[14] Meng, W., Ma, R. and Chen, H-H., 2014. "Smart Grid Neighborhood Area Networks: A Survey,” IEEE Network 28(1): 24-32, doi: 10.1109/MNET.2014.6724103.

[15] Mortaji, H., Ow, S., Moghavvemi, M., and Almurib, H., 2017. "Load Shedding and Smart-Direct Load Control Using Internet of Things in Smart Grid Demand Response Management", IEEE Transactions on Industry Applications 53(6), 5155-5163, doi: 10.1109/TIA.2017.2740832.

[16] Chang, K.H., 2013. "Interoperable nan standards: a path to cost-effective smart grid solutions," IEEE Wireless Communications 20(3): 4-5, doi: 10.1109/MWC.2013.6549274

[17] Al-Fuqaha, A., Guizani, M., Mohammadi, M., Aledhari, M., and Ayyash, M., 2015. "Internet of Things: A Survey on Enabling Technologies, Protocols and Applications," IEEE Communications Surveys \& Tutorials 17(4): 2015 23472376, doi:10.1109/COMST.2015.2444095.

[18] Luo, F., Zhao, J., Dong, Z.Y., Chen, Y., Xu, Y., Zhang, X., and Wong, K.P., 2016. "Cloud-based information infrastructure for next-generation power grid: Conception, architecture, and applications," IEEE Transactions on Smart Grid 7(4): 1896-1912, doi: 10.1109/TSG.2015.2452293.

[19] Lim, J., Doh, I., and Chae, K., 2017. "Secure and structured IoT smart grid system management", International Journal of Web and Grid Services 13(2): 170, doi: https://doi.org/10.1504/IJWGS.2017.083383.

[20] Chiu, W., Sun, H., Thompson, J., Nakayama, K., and Zhang, S., 2016. "Integrated communications, control, and computing technologies for enabling autonomous smart grid", IEEE Communications Magazine 54 (12): 58-59, doi: 10.1109/MCOM.2016.7786111

[21] Yang, C. Vyatkin, V. and Pang, C., "Service-oriented extension of IEC 61850 for model-driven smart grid automation design." in IECON 2017 - 43rd Annual Conference of the IEEE Industrial Electronics Society, 29 Oct-11 Nov 2017: 5489-5496, Bejing: IEEE.

[22] Carvalheira, E., and Coronel, E., 2014. "Testing the protection system in IEC 61850 communication-based substations." in IEEE ANDESCON, 15-17 Oct 2014: pp. 1-1, Cochabamba:IEEE.
[23] Li, Y., Cheng, X., Cao, Y., Wang, D., and Yang, L., 2018. "Smart Choice for the Smart Grid: Narrowband Internet of Things (NB-IoT)", IEEE Internet of Things Journal, 5(3): pp. 1505-1515, doi: 10.1109/JIOT.2017.2781251.

[24] Krata, J. and Saha, T., 2018. "Real-Time Coordinated Voltage Support with Battery Energy Storage in a Distribution Grid Equipped with Medium-Scale PV Generation", IEEE Transactions on Smart Grid: 1-1, doi: 10.1109/TSG.2018.2828991.

[25] Veldman, E., and Verzijlbergh, R., 2015. "Distribution Grid Impacts of Smart Electric Vehicle Charging from Different Perspectives", IEEE Transactions on Smart Grid 6(1): 333-342, doi: 10.1109/TSG.2014.2355494.

[26] Aburukba, R.O., Al-Ali, A., Landolsi, T., Rashid, M., and Hassan, R., 2016. "IoT based Energy Management for Residential Area," in IEEE International Conference on Consumer Electronics-Taiwan (ICCE-TW), 27-29 May 2016: 1-2, Taiwan:IEEE.

[27] Baimel, D., Tapuchi, S. and Baimel, N., 2016. "Smart Grid Communication Technologies", Journal of Power and Energy Engineering 4(8): 1-8, doi:10.4236/jpee.2016.48001

[28] Konstantelos, I., Giannelos, S., and Strbac, G., 2017. "Strategic valuation of smart grid technology options in distribution networks" IEEE Transactions on Power Systems 32(2): 1293-1303, doi: 10.1109/TPWRS.2016.2587999.

[29] Rossi, M., Viganò, G., Moneta, D., and Clerici, D., 2017. "Stochastic evaluation of distribution network hosting capacity: Evaluation of the benefits introduced by smart grid technology" in International Annual Conference, 20 22 Sep 2017:1-6, Cagiliari, Italy:IEEE.

[30] Wang, H., Qian, Y., and Sharif, H., 2013. "Multimedia communications over cognitive radio networks for smart grid applications," IEEE Wireless Communications 20 (4): 125-132, doi: 10.1109/MWC.2013.6590059.

[31] Cao, Z., Lin, J., Wan, C., Song, Y., Zhang, Y., and Wang, X., 2017. "Optimal cloud computing resource allocation for demand-side management," IEEE Transactions on Smart Grid, 8(4): 1943-1955, doi: 10.1109/TSG.2015.2512712.

[32] Cheng, X., Lee, W., and Pan, X., 2017. "Modernizing Substation Automation Systems: Adopting IEC Standard 61850 for Modeling and Communication". IEEE Industry Applications Magazine 23(1): 42-49, doi:10.1109/MIAS.2016.2600732.

[33] Miswan, N.S., Ridwan, M.I., Hayatudin, A., and Musa, I.A., 2015. "Interoperability testing for Digital Substation in Smart Grid domain: A power utility perspective" in International Symposium on Technology Management and Emerging Technologies (ISTMET), 25-27 Aug 2017: 154158, Langkawai Island:IEEE.

[34] Abe, R., Taoka, H., and McQuilkin, D., 2011. "Digital grid: communicative electrical grids of the future". IEEE Trans. Smart Grid 2(2): 399-410, doi: 10.1109/TSG.2011.2132744

[35] Deng, R., Yang, Z., Chow, M.-Y., and Chen, J., 2015. "A Survey on Demand Response in Smart Grids: Mathematical Models and Approaches," IEEE Transactions on Industrial Informatics 11 (3): 570- 582, doi: 10.1109/TII.2015.2414719.

[36] Shen, B., Yuan, Y., Xie, L., Zeng, X., Liu, Y., and Luo, H., 2017. "Reasch on IED configurator automatic modeling based on IEC 61850" in IEEE Conference on Energy Internet and Energy System Integration (EI2), 26-28 Nov $2017: 1-5$, Beijing:IEEE.

[37] Yang, Y., Xu, H., Gao, L., Yuan, Y., McLaughlin, K., and Sezer,K., 2017. "Multidimensional Intrusion Detection System for IEC 61850-Based SCADA Networks". IEEE Transactions on Power Delivery. Volume: 32 (2): 10681078, doi: 10.1109/TPWRD.2016.2603339.

[38] CRO "Renewable energy sources and climate change mtigation: special report of the Intergovernmental Panel on Climate Change", Choice Reviews Online, 49(11): 49-6309-49-6309, 2012, doi: https://www.ipcc.ch/report/renewableenergy-sources-and-climate-change-mitigation/

[39] Koko, S., Kusakana, K., and Vermaak, H., 2017. "Optimal energy management of a grid-connected micro-hydrokinetic with pumped hydro storage system", Journal of Energy Storage, 14: 8-15, doi: https://doi.org/10.1016/j.est.2017.09.007.

[40] Chena, W., Zhoua, K., Yanga, S., and Wuc, C., 2017. "Data quality of electricity consumption data in a smart grid environment", Renewable and Sustainable Energy Reviews, 75: 98-105, doi: https://doi.org/10.1016/j.rser.2016.10.054.

[41] Guerrero, J.L., García, A., Luque, J., and León, C., 2017. "Heterogeneous data source integration for smart grid ecosystems based on metadata mining", Expert Systems with Applications, 79: 254-268, doi: https://doi.org/10.1016/j.eswa.2017.03.007.

[42] Schett, G. 2017. "Digital substations for hydro power plants", in Proceedings of the Hydro Conference Series, Montreux, Switzerland 2017. 
[43] Torbaghan, M.E., Burrow, M., Hunt, D., and Elcheikh, M., 2017. "Risk-Based Framework (RBF) for a UK Pan-European Supergrid" Energy, 124: 124-132, doi: https://doi.org/10.1016/j.energy.2017.02.058.

[44] Einfalt, A., Cejka, S., Diwold, K., Frischenschlager, A., Faschang, M., Stefan, M., and Kupzog, F., 2017. "Interaction of smart grid applications supporting Plug \& Automate for intelligent secondary substations", CIRED - Open Access Proceedings Journal, 2017(1): 1257-1260, doi: 10.1049/oapcired.2017.1080.

[45] Viswanatham, V., Chari, A., and Saritha, V., 2016. "Region-based group and hierarchical key management for secure smart grid communications." International Journal of Smart Grid and Green Communications, 1(1): 50, doi: 10.1504/IJSGGC.2016.077289.

[46] La, Q., Chan, Y., and Soong, B., 2016. "Power Management of Intelligent Buildings Facilitated by Smart Grid: A Market Approach", IEEE Transactions on Smart Grid, 7(3): 1389-1400, doi: 10.1109/TSG.2015.2477852.

[47] Waleed, A., Zhang, Q., Tavakoli, M.M., Leung, S.F., Gu, L., He, J., and Fan, Z., 2016."Performance improvement of solution-processed CdS/CdTe solar cells with a thin compact TiO2 buffer layer", Science bulletin, 61(1): 86-91, doi: https://doi.org/10.1007/s11434-015-0963-0.

[48] Waleed, A., Hassan, K.M., and Virk, U.S., 2014. "Designing a dual axis solar tracker for optimum power", Journal of Electrical Engineering 14(2): 168173 , http://www.jee.ro/covers/art.php?issue=WH1375121304W51 f6af987b6d1.

[49] Waleed, A., and Fan, Z., 2017. "Fabrication of stable organometallic halide perovskite NWs based optoelectronic devices" Science bulletin, 62(9): 645647, doi: 10.1016/j.scib.2017.04.012.

[50] Tafreshi, S.M., and Lahiji, A.S., 2015. "Long-Term Market Equilibrium in Smart Grid Paradigm with Introducing Demand esponse Provider in Competition", IEEE Transactions on Smart Grid, 6(6): 2794-2806, doi: 10.1109/TSG.2015.2413951.

[51] Althaher, S., Mancarella, P., and Mutale, J., 2015. "Automated Demand Response from Home Energy Management System Under Dynamic Pricing and Power and Comfort Constraints", IEEE Transactions on Smart Grid, 6(4): 1874-1883, doi: 10.1109/TSG.2014.2388357.

[52] Kim, S-G., Hur, S-I, and Chae. Y-J., 2010. "Smart grid and its implications for electricity market design", Journal of Electrical Engineering and Technology, 5(1): 1-7, 2010.

[53] Sattarpour, T., Nazarpour, D., Golshannavaz, S., and Siano, P., 2015. "An optimal procedure for sizing and siting of DGs and smart meters in active distribution networks considering loss reduction", Journal of Electrical Engineering \& Technology, 10(3): 804-811, doi:10.5370/JEET.2015.10.3.804. 